\title{
Longitudinal dynamics of twin electron bunches in the Linac Coherent Light Source
}

\author{
Zhen Zhang, ${ }^{1,2}$ Yuantao Ding, ${ }^{1}$ Agostino Marinelli, ${ }^{1}$ and Zhirong Huang ${ }^{1}$ \\ ${ }^{1}$ SLAC National Accelerator Laboratory, Menlo Park, California 94025, USA \\ ${ }^{2}$ Department of Engineering Physics, Tsinghua University, Beijing 100084, China
}

(Received 18 December 2014; published 2 March 2015)

\begin{abstract}
The recent development of two-color x-ray free-electron lasers, as well as the successful demonstration of high-gradient witness bunch acceleration in a plasma, have generated strong interest in electron bunch trains, where two or more electron bunches are generated, accelerated and compressed in the same accelerating bucket. In this paper we analyze a twin-bunch technique in a high-energy linac, such as the Linac Coherent Light Source. This method allows the generation of two electron bunches with high peak current and independent control of time delay and energy separation. We find that the wakefields in the accelerator structures play an important role in the twin-bunch compression, and they can be used to extend the available time delay range. Based on the theoretical model and simulations we propose several methods to achieve larger time delay.
\end{abstract}

DOI: 10.1103/PhysRevSTAB.18.030702

PACS numbers: $41.75 . \mathrm{Ht}, 41.60 . \mathrm{Cr}$

\section{INTRODUCTION}

The advent of the x-ray free electron laser (XFELs) represents a revolution in light source development that opens up atomic imaging at femtosecond time scale. Despite the unequivocal success of existing XFELs [1-4], the growing user demands lead to continuing improvement of the facility capabilities. Two-color pulses are one of these capabilities developed under user requirements, where two pulses of different photoenergy and with a variable time delay are generated [5-9].

A recent experiment at the Linac Coherent Light Source (LCLS) [10] has shown the considerable advantages of driving a two-color XFEL with a train of two electron bunches of different energies. With respect to the standard single-bunch two-color methods, this twin-bunch technique allows to reach saturation for each bunch hence improves the FEL output by over 1 order of magnitude. It allows the timeresolved diagnostic of the FEL pulse with a deflecting cavity, and it can be coupled to the hard X-ray self-seeding system to generate two quasi-Fourier transform limited pulses.

Besides generating two-color XFELs, the twin-bunch scheme can also find its applications in the beam-driven plasma acceleration, e.g., the two-bunch experiments at FACET [11,12]. Compared with the masking technique adopted now [11], the twin-bunch method gives greater flexibility in the control of the charge distribution, peak current and time delay of the two bunches. This, in turn, leads to several advantages in the operation of plasma

Published by the American Physical Society under the terms of the Creative Commons Attribution 3.0 License. Further distribution of this work must maintain attribution to the author $(s)$ and the published article's title, journal citation, and DOI. wakefield accelerators since a time delay between the drive bunch and witness bunch provides a way to visualize the structure of the wakefields and settle down the witness bunch at the best accelerating phase.

The twin bunches can also be used in the fresh bunch scheme for the seeded free electron lasers to reduce the spectral noise [13]. Finally, the twin-bunch method has also been used in two-color Thomson scattering sources [14].

The generation of electron bunch trains in highbrightness photoinjectors has been extensively discussed in the scientific literature [15-17]. In this paper, we present comprehensive beam dynamic analysis of the twin-bunch method in a high-energy linac, including the control of the two pulses and the wakefield effects. We show that, thanks to the two-stage compression system, it is possible to control the time delay and energy separation of the two pulses independently. Wakefields play an important role in twin-bunch compression and can be used to obtain large time delay with methods proposed in the paper.

The paper is organized as follows. We begin in Sec. II with a detailed description and basic considerations of the twin-bunch method. In Sec. III, model analysis of the twinbunch compression is given without the linac wakefields to show the independent control of the time delay and energy separation. In Sec. IV we analyze the effects of the linac wakefields by theory and simulations. In Sec. V several methods are discussed with simulations to obtain large time delays. Finally, we give concluding remarks in Sec. VI.

\section{DESCRIPTION OF THE TWIN-BUNCH METHOD}

The twin-bunch method is schematically illustrated in Fig. 1. In a typical XFEL such as the LCLS, there are usually two magnetic chicanes as compressors in the 


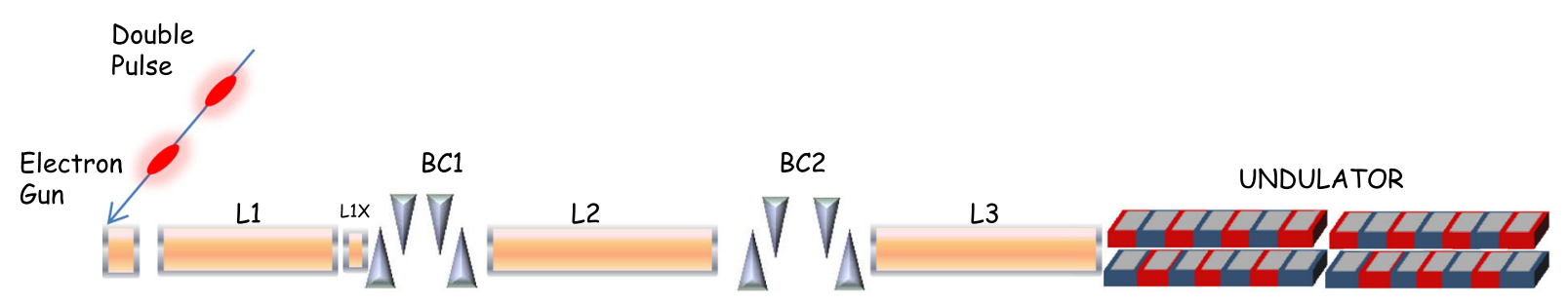

FIG. 1. A schematic layout of the two-bunch method to generate the two-color x-ray pulse (not to scale).

beam line ( $\mathrm{BC} 1$ and $\mathrm{BC} 2)$. The electrons are generated by a photocathode illuminated by a train of two laser pulses with a variable delay on the order of a few picoseconds. Note that this is different from the multibunch method in [18] in separate radio-frequency (rf) cycles. The two bunches are accelerated off-crest in the linear accelerators (L1 and L2) and compressed by the two chicanes to increase the peak current by a factor of $\sim 200$. This process generates an energy difference between the two bunches due to the off-crest acceleration. In addition, the arriving time difference will be compressed to the order of a few tens of femtoseconds.

Compared to the single bunch compression, twin-bunch compression has to achieve several goals at the same time: (1) control the time delay (2) control the energy separation, (3) obtain high peak current and (4) control the remaining energy chirp (the latter is important to control the spectral properties of self-seeded XFELs [19,20]). Usually these goals are coupled together. In the configuration of Fig. 1, there are several knobs to control the twin-bunch compression: initial time delay after the injector, off-crest phases of L1 and L2, and longitudinal dispersion $R_{56}$ of $\mathrm{BC} 1$ and $\mathrm{BC}$. The high-harmonic cavity (L1X) before $\mathrm{BC} 1$ can also be used to optimize the energy chirp. Note that when we change the phase of L1 and L2, the rf amplitude of these two linac sections will be adjusted so the energy gain is kept constant. In the practical implementation of the two-bunch scheme, we usually choose to fix the $R_{56}$ of the two chicanes and vary other knobs.

\section{ANALYSIS OF THE TWIN-BUNCH COMPRESSION WITHOUT WAKEFIELDS}

In this section we study a simplified model of the longitudinal beam dynamics of the twin-bunch compression which does not include linac wakefields. For the longitudinal phase space $(z, \delta)$, where $z$ is the longitudinal coordinate with head bunch at $z<0$ and $\delta$ is the relative energy spread, the transfer matrix of each section of the beam line in Fig. 1 can be written as

$$
\begin{array}{ll}
M_{L 1}=\left(\begin{array}{cc}
1 & 0 \\
h_{1} & \frac{E_{0}}{E_{1}}
\end{array}\right), & M_{B C 1}=\left(\begin{array}{cc}
1 & R_{56}^{(1)} \\
0 & 1
\end{array}\right), \\
M_{L 2}=\left(\begin{array}{cc}
1 & 0 \\
h_{2} & \frac{E_{1}}{E_{2}}
\end{array}\right), & M_{B C 2}=\left(\begin{array}{cc}
1 & R_{56}^{(2)} \\
0 & 1
\end{array}\right),
\end{array}
$$

where $E_{0}, E_{1}, E_{2}$ are the beam average energies at the entrance of $\mathrm{L} 1, \mathrm{BC} 1$ and $\mathrm{BC} 2$, respectively. $h_{1}, h_{2}$ denote the energy chirp added by the off-crest acceleration in the linac and $h_{1}=-\frac{k\left(E_{1}-E_{0}\right) \tan \left(\phi_{1}\right)}{E_{1}}, h_{2}=-\frac{k\left(E_{2}-E_{1}\right) \tan \left(\phi_{2}\right)}{E_{2}}$ with $\phi_{1}, \phi_{2}$ being the phases of L1 and L2 and $k$ being wave number. The transfer matrix for the whole beam line can be obtained by multiplying all sections together:

$$
M=M_{L 3} M_{B C 2} M_{L 2} M_{B C 1} M_{L 1}=\left(\begin{array}{cc}
1+R_{56}^{(1)} h_{1}+R_{56}^{(2)}\left(h_{2}+h_{1} \frac{E_{1}}{E_{2}}+R_{56}^{(1)} h_{1} h_{2}\right) & R_{56}^{(1)} \frac{E_{0}}{E_{1}}+R_{56}^{(2)}\left(R_{56}^{(1)} \frac{E_{0}}{E_{1}} h_{2}+\frac{E_{0}}{E_{2}}\right) \\
\frac{E_{2}}{E_{3}}\left(h_{2}+h_{1} \frac{E_{1}}{E_{2}}+R_{56}^{(1)} h_{1} h_{2}\right) & R_{56}^{(1)} h_{2} \frac{E_{0} E_{2}}{E_{1} E_{3}}+\frac{E_{0}}{E_{3}}
\end{array}\right)
$$

Here $E_{3}$ is the final beam energy. We assume the third linac section L3 accelerates the beam on-crest and there is no energy chirp added on the beam. The energy separation at the exit of the photoinjector can be neglected and the longitudinal separation is defined as the average longitudinal coordinate of the tail bunch minus the one of the head bunch. Then the final longitudinal separation and energy difference of the two bunches can be obtained by the transfer matrix:

$$
\Delta z=\left[1+R_{56}^{(1)} h_{1}+R_{56}^{(2)}\left(h_{2}+h_{1} \frac{E_{1}}{E_{2}}+R_{56}^{(1)} h_{1} h_{2}\right)\right] \Delta z_{0},
$$

$$
\delta=\left(h_{2}+h_{1} \frac{E_{1}}{E_{2}}+R_{56}^{(1)} h_{1} h_{2}\right) \frac{E_{2}}{E_{3}} \Delta z_{0} .
$$

We use the LCLS beam line parameters as an example, which are shown in Table I. The initial time delay of the two bunches can be varied from 5 to 9 ps. It is known that the beam transverse emittance depends on the emission phase at the cathode [21,22] and varying time delay will also change emittance. So it is undesirable to vary the time delay frequently. In the following analysis, for simplicity, we fix it at 6 ps. Figure 2 shows the contours of the time delay and relative energy separation, calculated by Eqs. (3) 
TABLE I. Beam line parameters of the LCLS for the twinbunch compression.

\begin{tabular}{lcc}
\hline \hline Parameter & Value & Units \\
\hline Total charge $Q$ & 150 & $\mathrm{pC}$ \\
Initial peak current $I_{p}$ & 25 & $\mathrm{~A}$ \\
Initial time delay $\tau_{0}$ & $6(5-9)$ & $\mathrm{ps}$ \\
L1 phase $\phi_{1}$ & $-20 \sim-40$ & $\mathrm{deg}$ \\
L2 phase $\phi_{2}$ & $-30 \sim-40$ & $\mathrm{deg}$ \\
L1X amplitude & $0-22$ & $\mathrm{MV}$ \\
L1X phase & -160 & $\mathrm{deg}$ \\
Injector energy $E_{0}$ & 135 & $\mathrm{MeV}$ \\
BC1 energy $E_{1}$ & 220 & $\mathrm{MeV}$ \\
BC2 energy $E_{2}$ & 5 & $\mathrm{GeV}$ \\
Final energy $E_{3}$ & 13 & $\mathrm{GeV}$ \\
$R_{56}$ of BC1 & -45.5 & $\mathrm{~mm}$ \\
$R_{56}$ of BC2 & -24.7 & $\mathrm{~mm}$ \\
\hline \hline
\end{tabular}

and (4). Simulation results by LiTRACK [23] without the linac wakefields are also given in Fig. 2. LiTRACK is a 1D simulation code, which only tracks the longitudinal phase space. The transverse beam dynamics and collective effects except the longitudinal wakefields are fully neglected.
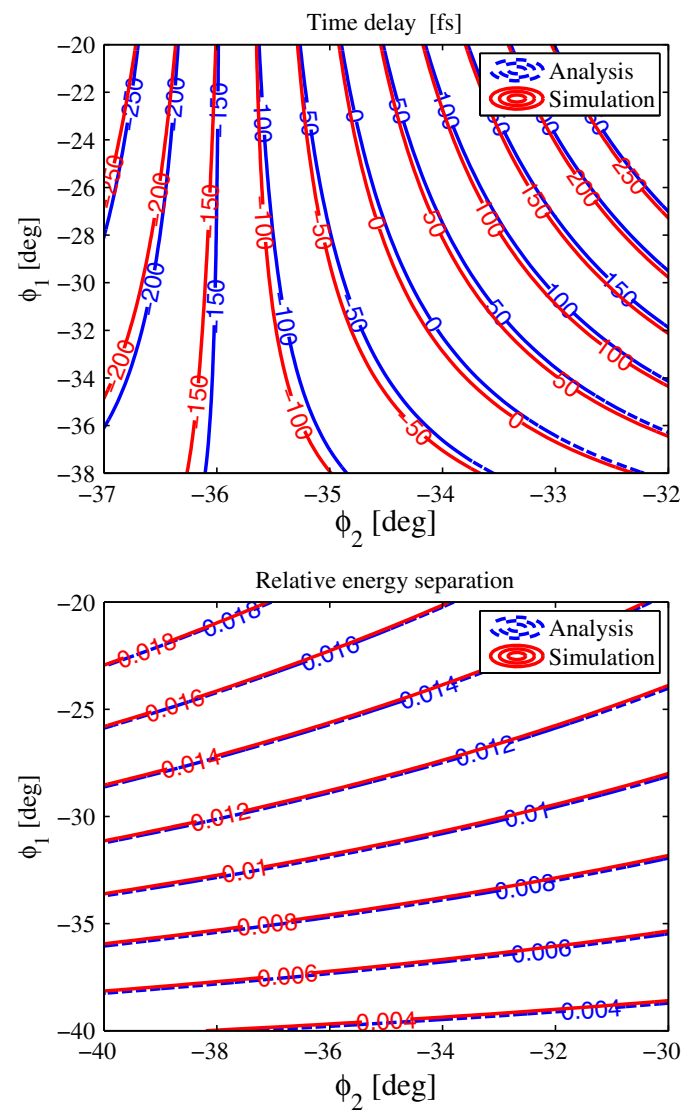

FIG. 2. The contours of the time delay (up) and relative energy separation (down) of the two bunches. The blue dashed line is the analysis results of Eqs. (3) and (4) and the red line is the simulation results of LITRACK without the linac wakefields. The amplitude of L1X in this simulation is set to be zero.
Note that, in this analysis, we are neglecting nonlinear terms in the energy chirp. This approximation implicity takes into account the effect of the harmonic linearizing cavity, which removes the second order time-energy correlation induced by the injector and L1.

The analysis results and the LITRACK simulations agree well except for a small deviation in the time delay, which is due to the nonlinear effects in the magnetic compression. With the above definitions, a time delay means that the tail bunch has surpassed the head bunch during the compression process. The contour curves in Fig. 2 imply a feature of the twin-bunch method. For the time delay. especially in the range from -250 to $50 \mathrm{fs}$, it mostly depends on $\phi_{2}$. As for the energy separation, it is mostly determined by $\phi_{1}$. In other words, we can control the time and energy dimensions independently. This feature can be understood by the analytical results. We take derivatives with respect to $h_{1}$ and $h_{2}$ in Eqs. (3) and (4),

$$
\begin{gathered}
\frac{\partial \Delta z}{\partial h_{1}}=\left(R_{56}^{(1)}\left(1+R_{56}^{(2)} h_{2}\right)+R_{56}^{(2)} \frac{E_{1}}{E_{2}}\right) \Delta z_{0}, \\
\frac{\partial \Delta z}{\partial h_{2}}=\left(1+R_{56}^{(1)} h_{1}\right) R_{56}^{(2)} \Delta z_{0}, \\
\frac{\partial \delta}{\partial h_{1}}=\left(R_{56}^{(1)} h_{2}+\frac{E_{1}}{E_{2}}\right) \frac{E_{2}}{E_{3}} \Delta z_{0}, \\
\frac{\partial \delta}{\partial h_{2}}=\left(1+R_{56}^{(1)} h_{1}\right) \frac{E_{2}}{E_{3}} \Delta z_{0} .
\end{gathered}
$$

When $\left|1+h_{2} R_{56}^{(2)}\right| \approx 0$ and $\frac{E_{1}}{E_{2}} \ll\left|1+h_{1} R_{56}^{(1)}\right| \ll\left|h_{2} R_{56}^{(1)}\right|$, we will have

$$
\begin{gathered}
\left|\frac{\partial \Delta z}{\partial h_{2}}\right| \gg\left|\frac{\partial \Delta z}{\partial h_{1}}\right|, \\
\left|\frac{\partial \delta}{\partial h_{1}}\right| \gg\left|\frac{\partial \delta}{\partial h_{2}}\right| .
\end{gathered}
$$

Thus, the time delay and energy separation are determined by the phases of L2 and L1, respectively. The first condition means $\mathrm{BC} 2$ works at around full compression and the second is satisfied well under the parameters of LCLS in Table I. The independent control of time delay and energy separation in the twin-bunch scheme has been confirmed in the experiment at the LCLS [10].

\section{WAKEFIELD EFFECT IN THE TWIN-BUNCH COMPRESSION}

The longitudinal wakefields of the linac can induce longitudinal-dependent energy loss, which have significant effects on the twin-bunch compression. If this happens before the dispersion section, for example the wakefields of 
L2, the time delay and energy separation will be changed. For the wakefield after the final chicane, e.g., in L3, it will only change the energy separation. So our analysis will focus on the wakefields before the chicane.

The wake-induced relative energy loss at the longitudinal coordinate $z$ is (see. e.g., Refs. [24,25])

$$
\delta_{w}(z)=-\frac{e^{2} L}{\gamma m c^{2}} \int_{0}^{\infty} W(z) n\left(z-z^{\prime}\right) d z^{\prime},
$$

with $W(z)$ the point charge wake function, $c$ the speed of light, $\gamma$ the Lorentz factor, $L$ the length of the linac and $n(z)$ the longitudinal bunch distribution normalized as $\int_{-\infty}^{\infty} n(z) d z=N$ ( $N$ is the total number of the electrons in the bunch). Consider the condition

$$
\frac{a^{2}}{2 L} \ll \sigma_{z} \ll s_{0}
$$

where $a$ is the pipe radius, $\sigma_{z}$ the bunch length and $s_{0}$ the characteristic length of the wakefields. The first inequality says that the length of structure $L$ is much larger than the catch-up distance and the transient behavior of the wake can be ignored. Under this condition, we can use the limiting value of the steady-state wake for periodic structure,

$$
W\left(0^{+}\right)=\frac{Z_{0} c}{\pi a^{2}},
$$

where $Z_{0}=377 \Omega$. Inserting Eq. (13) into Eq. (11), we obtain

$$
\delta_{w}(z)=-\frac{4 r_{e} L}{\gamma a^{2}} \int_{0}^{\infty} n\left(z-z^{\prime}\right) d z^{\prime}
$$

with the classical electron radius $r_{e}=2.82 \times 10^{-15} \mathrm{~m}$. For simplicity, we assume that the twin bunches have a uniform current profile with full bunch length $L_{b}$ and longitudinal separation $T$, i.e.,

$$
n(z)=n_{0} \begin{cases}\frac{T}{2 L_{b}} & \left|z \pm \frac{T}{2}\right| \leq \frac{L_{b}}{2}, \\ 0 & \text { otherwise }\end{cases}
$$

Here $n_{0}=\frac{N}{T}$ and we define the "duty factor" as

$$
\eta=\frac{L_{b}}{T}
$$

to represent the structure of the twin bunches with $0<\eta<1$ (no overlap between the two bunches). Inserting Eq. (15) into Eq. (14), we can get the wakeinduced energy loss due to the wakefields as a function of the beam longitudinal coordinate $z$ :

$$
\delta_{w}(z)=-\delta_{0} \begin{cases}0 & z<-\frac{T}{2}-\frac{L_{b}}{2}, \\ \frac{1}{2 L_{b}}\left(z+\frac{T}{2}+\frac{L_{b}}{2}\right) & \left|z+\frac{T}{2}\right| \leq \frac{L_{b}}{2}, \\ \frac{1}{2} & |z|<\frac{T}{2}-\frac{L_{b}}{2}, \\ \frac{1}{2}+\frac{1}{2 L_{b}}\left(z-\frac{T}{2}+\frac{L_{b}}{2}\right) & \left|z-\frac{T}{2}\right| \leq \frac{L_{b}}{2}, \\ 1 & z>\frac{T}{2}+\frac{L_{b}}{2},\end{cases}
$$

where $\delta_{0}=\frac{4 r_{e} L N}{\gamma a^{2}}$. The beam longitudinal distribution and the relative energy loss are given in Fig. 3. The stairlike distribution of energy loss indicates that the effects of the wakefields can be divided into two types. One is changing the average energy of the two bunches (as shown in magenta dashed line in the middle of Fig. 3) and the other is changing the energy chirp of each bunch. This can be understood better by rewriting the form of energy loss. Within the longitudinal coordinate of the two bunches $\left(\left|z \pm \frac{T}{2}\right| \leq \frac{L_{b}}{2}\right)$, the energy loss can be rewritten as

$$
\begin{aligned}
\delta_{w}(z) & =-\frac{\delta_{0}}{2 T} z-\frac{\delta_{0}}{2 L_{b}}(1-\eta)\left(z \pm \frac{T}{2}\right) \\
& =\delta_{w}^{G}(z)+\delta_{w}^{L}(z) .
\end{aligned}
$$

The sign \pm corresponds to the positions of the two bunches. The first term on the right side of Eq. (18), $\delta_{w}^{G}(z)$, is an energy chirp over the whole bunch, which is similar with the rf-induced chirp. We refer to this kind of chirp as "global energy chirp." The wake-induced global energy chirp can be compensated by adjusting the phase of the linac sections. The second terms, $\delta_{w}^{L}(z)$, induce "local
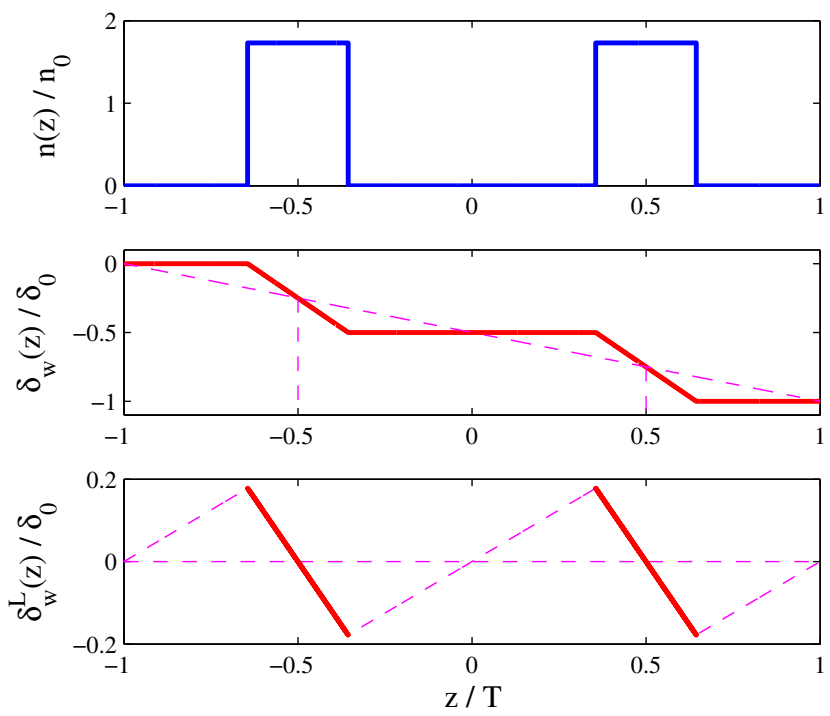

FIG. 3. (Top) beam longitudinal distribution $n(z)$, (middle) relative energy loss due to the wakefields $\delta_{w}(z)$ and (down) relative energy loss after compensating the energy change of the two bunch centers by adjusting the rf chirp. Here we adopt $\eta=\frac{\sqrt{3}}{6}$. 
energy chirp" with $\delta_{w}^{L}\left(z= \pm \frac{T}{2}\right)=0$. The distribution of $\delta_{w}^{L}(z)$ is also given in the lower plot of Fig. 3. Taking the derivatives with respect to $z$ in Eq. (18), we can get the slope of the energy chirp:

$$
\begin{aligned}
\frac{d \delta_{w}(z)}{d z} & =-\frac{\delta_{0}}{2 T}-\frac{\delta_{0}}{2 L_{b}}(1-\eta) \\
& =\frac{d \delta_{w}^{G}(z)}{d z}+\frac{d \delta_{w}^{L}(z)}{d z} .
\end{aligned}
$$

The slope of the rf energy chirp in L2 is $h_{2} \approx-k \tan \left(\phi_{2}\right)$ when $E_{2} \gg E_{1}$. So in order to compensate $\delta_{w}^{G}(z), \phi_{2}$ needs to be adjusted by

$$
\Delta \phi_{2} \approx-\frac{\delta_{0} \cos ^{2}\left(\phi_{2}\right)}{2 k T} .
$$

For the local energy chirp, since $0<\eta<1$, the slope is always negative, which will weaken the beam compression. We define the compression factor as

$$
|C|=\left|\frac{1}{1+h_{L} R_{56}}\right|=\frac{I_{f}}{I_{i}},
$$

where $h_{L}$ is the slope of local energy chirp and $I_{i}, I_{f}$ the peak current before and after the compression. $C>0(<0)$ means under (over) compression. The slope of the global energy chirp can be obtained as

$$
h_{G}=h_{L}-\frac{d \delta_{w}^{L}}{d z} .
$$

With these definitions and approximations, we can get an analytical expression for the final time delay for a single bunch compressor:

$$
\Delta T=\frac{T}{C}+\frac{\delta_{0}}{2}\left(\frac{1}{\eta}-1\right) R_{56} .
$$

For the double chicane compression system, we further assume that the wakefields before $\mathrm{BC} 1$ are negligible, and we get the equation for the final time delay by replacing $T$ by $\frac{\tau_{0}}{C_{1}}$,

$$
\Delta T=\frac{\tau_{0}}{C_{1} C}+\frac{2 r_{e} L N}{\gamma a^{2}}\left(\frac{1}{\eta}-1\right) R_{56}^{(2)},
$$

where $C_{1}=\frac{1}{1+h_{1} R_{56}^{(1)}}$ is the compression factor of $\mathrm{BC} 1$. Since $\tau_{0}>0, \eta<1$ and $R_{56}<0$, the second term on the right side, named "wake-induced term," is always negative and the first term, "compression term," can be either, depending on the compression of the local energy chirp.
The value of the final time delay is the sum of these two terms.

Note that the condition to use the limiting value of the steady-state wake requires $\sigma_{z} \ll s_{0}$ and $s_{0}=1.5 \mathrm{~mm}$ for the SLAC S-band structure [24]. The bunch length after the injector is $\sim 6 \mathrm{ps}$ and the compression factor of $\mathrm{BC} 1$ is $\sim 5$. So the whole bunch length is usually $\sim 0.4 \mathrm{~mm}$ in L2 and the requirement of "much smaller" is not satisfied well. The resulting distribution of the energy loss induced by the wakefields is not a perfect stairlike shape, but it has nonlinear terms. However, these nonlinear wakes have little effect on the global properties of the twin bunches, such as their time delay and energy separation and can be neglected in this simplified analysis.

Figure 4 shows a schematic representation of the effect of wakefields on the time delay. If we neglect the effect of wakefields, the time delay is zero when both bunches are close to full compression (i.e., maximum peak current) since both bunches are accelerated at roughly the same phase. If we include wakefields, the physical picture changes significantly. Since the wakefields have a stairlike shape, the local energy chirp is smaller than the average energy chirp of the twin-bunch system. This means that the compression curve of the individual bunches in the lower plot of Fig. 4 is shifted to the right and the two bunches reach the maximum peak current at a delay different than 0 .

Figure 5 shows the contours of time delay and energy separation with linac wakefields in the LITRACK simulation. After including wakefields, the time delay is shifted left by $\sim 50$ fs along $\phi_{2}$, which confirms that the wakeinduced global energy chirp can be compensated by tuning the phase of the linac. The change of the energy separation contour can be divided into two conditions. When BC2 works at undercompression, the wakefields will decrease the energy separation. At overcompression, the effect will be inverse. This is because at overcompression the two
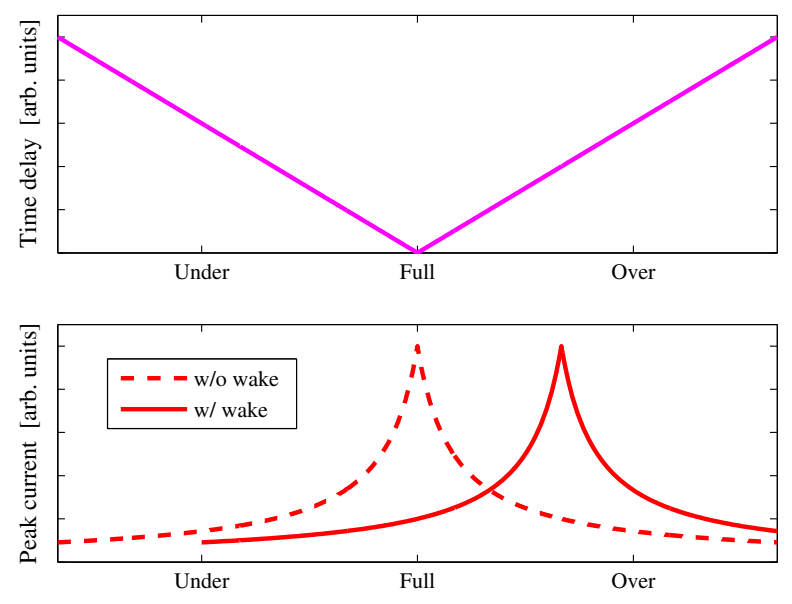

FIG. 4. A schematic representation of time delay (up) and peak current (down) versus the compression mode. The dashed line is without the wakes and the solid line is with the wakes. 

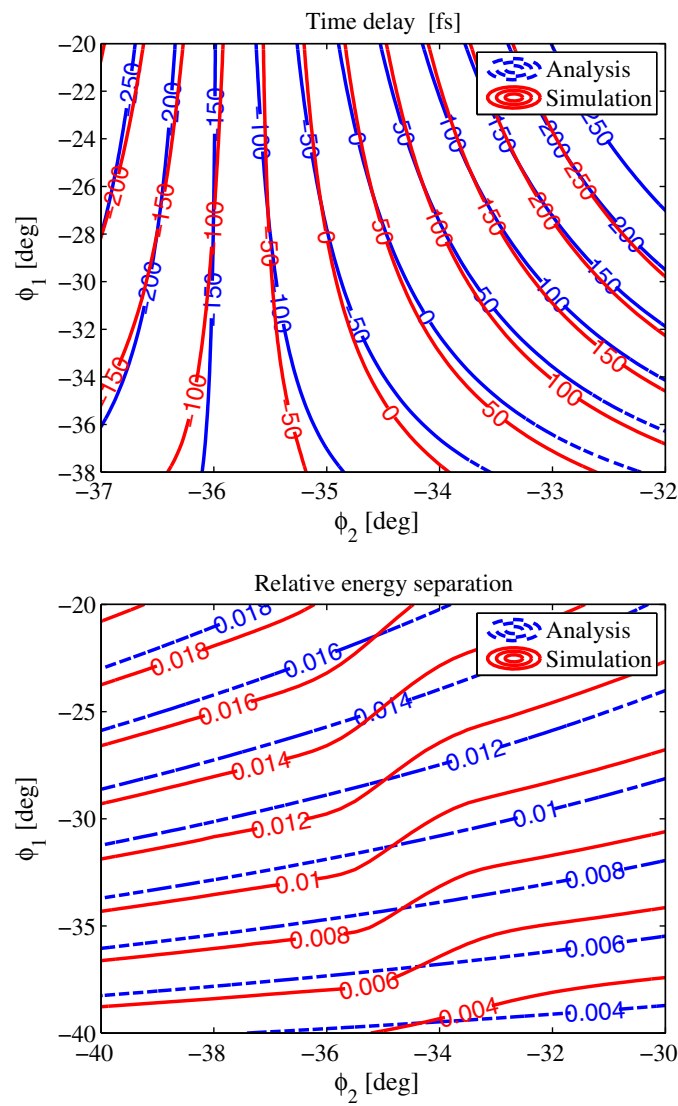

FIG. 5. The contours of the time delay (up) and relative energy separation (down) of the two bunches. The blue dashed line is the analysis results of Eqs. (3) and (4) and the red line is the simulation results of LITRACK with the linac wakefields. The amplitude of L1X in this simulation is set to be zero.

bunches exchange their longitudinal order in L3 (the tail bunch comes first).

We now discuss the optimization of the twin-bunch compression for the LCLS using the LITRACK particle tracking code. We will use the beam parameters in Table I. Note that, in addition to the time delay and energy separation, peak current is another important parameter of the twin bunches. For example, when we apply this method to generate two-color hard $\mathrm{x}$ rays in an FEL, the peak current needs to be $\sim 4 \mathrm{kA}$ to acquire a reasonable gain length.

To optimize the twin-bunch compression we run LITRACK simulations scanning the L1X amplitude and the phases of L1 and L2. From the scan results, we pick out "working points" with suitable peak current and plot their energy separation and time delay, as shown in Fig. 6. Figure 6 also shows three typical phase spaces.

From Fig. 6 it is clear that the peak current requirements limit the available ranges of time delay and energy separation. Fox example, when the energy separation is $0.5 \%$, the time delay is $<150 \mathrm{fs}$. It is also noticeable that the area of working points has two branches. This can be
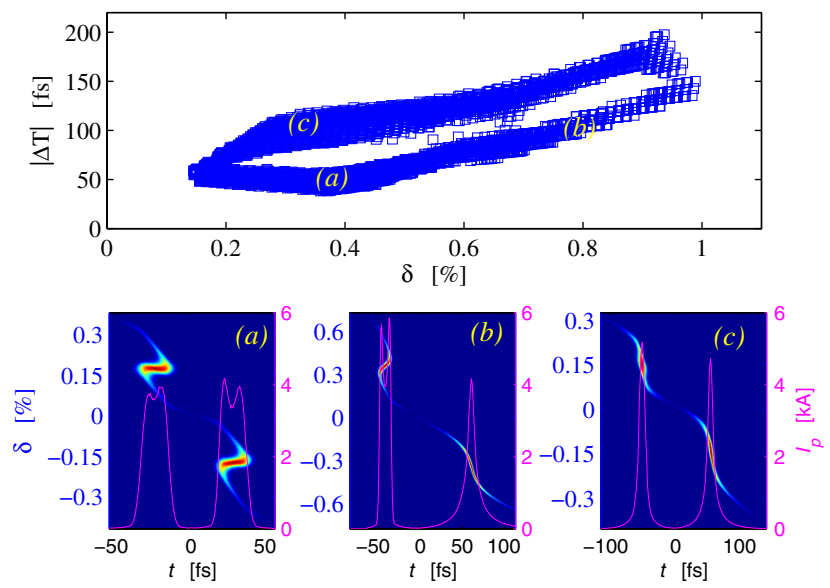

FIG. 6. Working points with peak current 3-5 kA of the core part of the beam (up) and three typical longitudinal phase spaces with current profiles (down) of the twin bunches at the exit of L3, which are labeled $(a),(b),(c)$ respectively.

understood by Eq. (24). For a fixed peak current, the compression factor can be either positive or negative, corresponding to under- or overcompression of the individual bunches. In the first case, the final time delay is the subtraction of the two terms in Eq. (24), corresponding to the lower branch, while the other branch corresponds to two overcompressed bunches. Note how the upper branch gives larger time delay with similar energy separation of the lower branch. Figures 6(a) and 6(c) show typical phase spaces for two undercompressed and overcompressed bunches. The main change in the beam line settings in Table II from $(a)$ to $(c)$ is the phase of L2 with the overcompressed case corresponding to a more negative phase.

As the energy separation increases, the nonlinear components of the L1 accelerating field become non-negligible. This can result in the two bunches being compressed to two opposite regimes, with the low energy bunch being overcompressed and the high-energy one undercompressed [see in Fig. 6(b)]. This case is not well described by our theoretical model which only includes the linear term in the rf field.

The difference of rf energy chirp on the twin bunches can be compensated, at least partly, by increasing the amplitude and optimizing the phase of L1X. Moreover, we assumed the same initial peak current for the twin bunches in the previous analysis. If we put more charge in the head bunch,

TABLE II. The beam line parameters for the three typical phase spaces in Fig. 6.

\begin{tabular}{lccc}
\hline \hline & Beam $(a)$ & Beam $(b)$ & Beam $(c)$ \\
\hline L1 phase (deg) & -25.4 & -21.0 & -25.5 \\
L2 phase (deg) & -35.7 & -36.5 & -39.4 \\
L1X amplitude (MV) & 20 & 19 & 21 \\
\hline \hline
\end{tabular}


the wake-induced local energy chirp will also help compensate the nonlinear components of the rf energy chirp.

\section{METHODS TO CONTROL THE TIME DELAY}

We have shown before that the time delay of the two bunches is strongly affected by longitudinal wakefields. In particular, we have identified a wake-induced term in the delay that depends on a few independent parameters such as the beam energy at $\mathrm{BC} 2$ and the $\mathrm{BC} 2 \mathrm{R} 56$.

From Eq. (24) follows that we have several choices to increase the wakefield-induced delay: reducing the beam energy of $\mathrm{BC} 2$; increasing the amplitude of the wakes; decreasing the duty factor; increase bunch charge and increasing the value of $R_{56}^{(2)}$. However, the initial bunch charge and duty factor are limited by other considerations, e.g., maintaining the beam emittance in the injector. In what follows we will be concerned with the other three parameters.

The first method is to reduce the beam energy of $\mathrm{BC} 2$. Here we vary it from 5 to $3 \mathrm{GeV}$ and scan other variable parameters (the energy loss due to reducing $\mathrm{BC} 2$ energy is compensated by L3 and the final beam energy will be kept the same). As in the previous section, we select working points for which the peak current of both bunches is in the range $3 \mathrm{kA}<I<5 \mathrm{kA}$ and plot them in Fig. 7. We separate the working points into two groups corresponding to the tail bunch being over- or undercompressed. The bluesquare points are the results with the nominal beam energy of $5 \mathrm{GeV}$. Reducing the $\mathrm{BC} 2$ beam energy moves the area of suitable working points upwards, which means larger time delay under the same energy separation.

The second method is to increase the $R_{56}$ of $\mathrm{BC} 2$. The nominal $\mathrm{BC} 2 R_{56}$ for $5 \mathrm{GeV}$ electron beam is $-24.7 \mathrm{~mm}$. In our study, we increase the $R_{56}$ by $20 \%$ to $50 \%$. We also separate them into two groups and plot with time delay and energy separation as shown in Fig. 8. Increasing $R_{56}$ of $\mathrm{BC} 2$ also shifts the area of working points upwards and generates larger time delay.

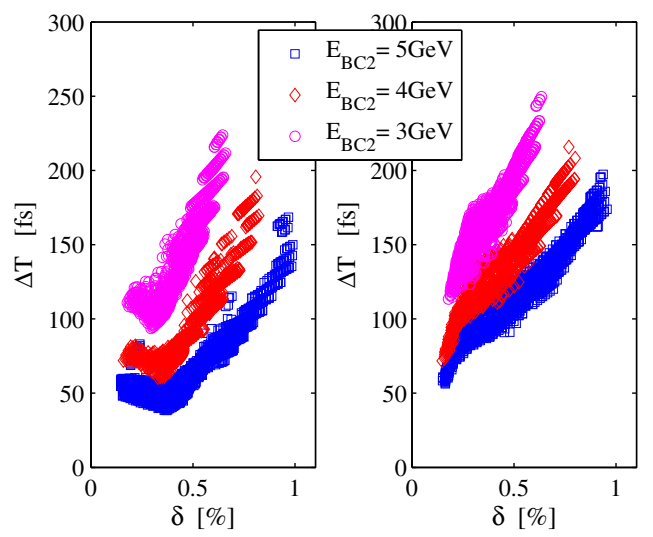

FIG. 7. Working points for different beam energy of BC2. The tail bunch is under (left) and over (right) compression, respectively. The peak current requirement is $3-5 \mathrm{kA}$ of the core beam.

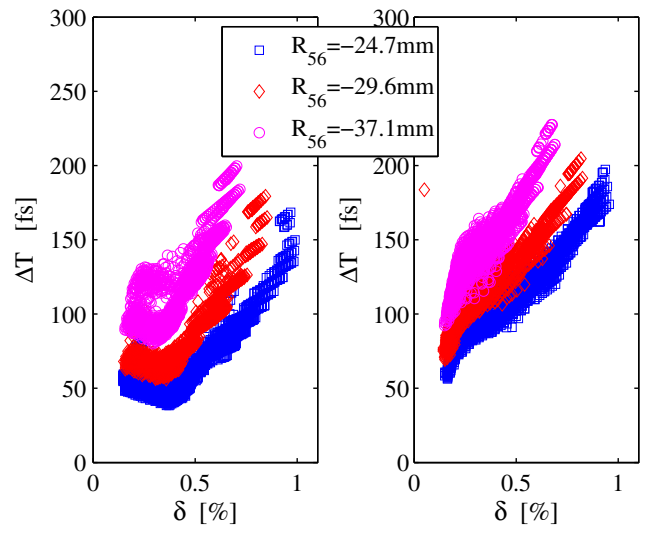

FIG. 8. Working points for different $R_{56}$ of BC2. The tail bunch is under (left) and over (right) compression, respectively. The peak current requirement is $3-5 \mathrm{kA}$ of the core beam.

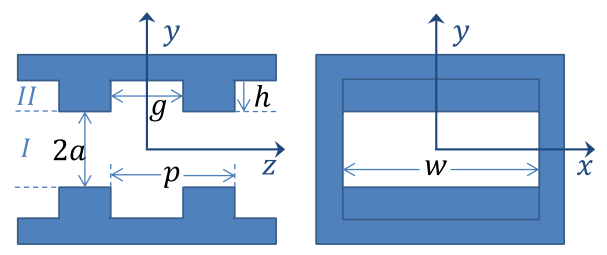

FIG. 9. Geometry of dechirper parameters: longitudinal cut with two periods (left) and transverse cut (right).

The third method is to increase the wakefield before $\mathrm{BC} 2$. This can be achieved by adding a rectangular corrugated structure in the beam line, a device known as a passive dechirper [26,27]. This device was originally developed to control the beam energy chirp in linacs $[28,29]$. The geometry of the device is shown in Fig. 9. The parameters of the structure used here are given in Table III. We can set different values of the aperture $a$ to vary the wakefields $(a=\infty$ corresponds to an open structure). For a given $a$, we scan all variable beam line parameters and plot working points out in Fig. 10. The amplitude of the longitudinal wakefield is inverse proportional to $a^{2}$. As the dechirper wakefield intensity increases (i.e., for small values of $a$ ), the area of suitable working points will move towards the up-right corner-reaching larger time delay and larger energy separation.

From the above analysis we can summarize the three methods together in Fig. 11. The solid ellipse denotes the

TABLE III. Parameters of the corrugated structure.

\begin{tabular}{lcc}
\hline \hline Parameter & Value & Units \\
\hline Half-gap $a$ & $>2$ & $\mathrm{~mm}$ \\
Period $p$ & 1 & $\mathrm{~mm}$ \\
Depth $h$ & 1 & $\mathrm{~mm}$ \\
Opening $g$ & 0.5 & $\mathrm{~mm}$ \\
Width $w$ & 24 & $\mathrm{~mm}$ \\
Length $L$ & 10 & $\mathrm{~m}$ \\
\hline \hline
\end{tabular}




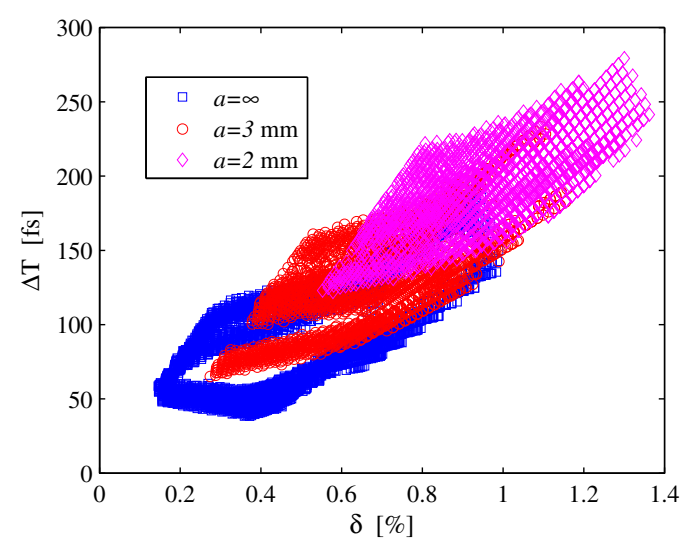

FIG. 10. Working points for different $a$ of the wakefield structure. The peak current requirement is $3-5 \mathrm{kA}$ of the core beam.

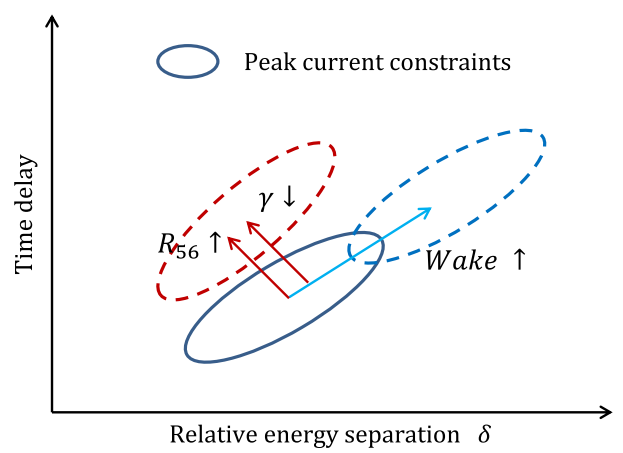

FIG. 11. Schematic of the three methods to acquire large time delay for the two-bunch compression scheme.

working points. Reducing the beam energy and increasing the $R_{56}$ of the dispersion both move the ellipse upwards. Increasing wakefield before $\mathrm{BC} 2$ will move the ellipse upright. All these three methods can help extend the available range of the time delay and we can combine them together to optimize the performance of the twin-bunch method.

\section{CONCLUSIONS}

In this paper we studied the longitudinal beam dynamics of generating high-energy and high-intensity twin bunches. The beam dynamics of twin bunches is of great relevance for many fields, such as two-color XFELs, seeded FELs and beam-driven plasma acceleration. Our analysis shows that the final time delay and energy separation of the twin bunches can be controlled independently while keeping the peak current of the two bunches within a suitable range. We have shown that wakefields have a strong influence on the final time delay of the two bunches and their effect can be used to our advantage in controlling the twin-bunch dynamics. Based on our theoretical analysis, we proposed three methods to vary the time delay by controlling the effect of wakefields.

\section{ACKNOWLEDGMENTS}

This work was supported under U.S. Department of Energy Contract No. DE-AC02-76SF00515.

[1] W. Ackermann et al., Nat. Photonics 1, 336 (2007).

[2] P. Emma et al., Nat. Photonics 4, 641 (2010).

[3] T. Ishikawa et al., Nat. Photonics 6, 540 (2012).

[4] E. Allaria et al., Nat. Photonics 6, 699 (2012).

[5] A. Lutman, R. Coffee, Y. Ding, Z. Huang, J. Krzywinski, T. Maxwell, M. Messerschmidt, and H.-D. Nuhn, Phys. Rev. Lett. 110, 134801 (2013).

[6] G. De Ninno, B. Mahieu, E. Allaria, L. Giannessi, and S. Spampinati, Phys. Rev. Lett. 110, 064801 (2013).

[7] V. Petrillo et al., Phys. Rev. Lett. 111, 114802 (2013).

[8] T. Hara et al., Nat. Commun. 4, 2919 (2013).

[9] A. Marinelli, A. A. Lutman, J. Wu, Y. Ding, J. Krzywinski, H.-D. Nuhn, Y. Feng, R. N. Coffee, and C. Pellegrini, Phys. Rev. Lett. 111, 134801 (2013).

[10] A. Marinelli et al., Nat. Commun. 6, 6369 (2015).

[11] M. J. Hogan et al., New J. Phys. 12, 055030 (2010).

[12] M. Litos et al., Nature (London) 515, 92 (2014).

[13] L. H. Yu, in Mini-workshop on present and future FEL schemes, 2008, Shanghai.

[14] V. Petrillo et al., Phys. Rev. ST Accel. Beams 17, 020706 (2014).

[15] M. Ferrario et al., Nucl. Instrum. Methods Phys. Res., Sect. A 637 S43 (2011).

[16] P. Musumeci, R. K. Li, and A. Marinelli, Phys. Rev. Lett. 106, 184801 (2011).

[17] C. Ronsivalle et al., New J. Phys. 16, 033018 (2014).

[18] F.-J. Decker et al., in Proceedings of the 32nd Free Electron Laser Conference, Malmö, Sweden (Max-lab, Sweden, 2010).

[19] J. Amann et al., Nat. Photonics 6, 693 (2012).

[20] A. A. Lutman et al., Phys. Rev. Lett. 113, 254801 (2014).

[21] B. E. Carlsten, Nucl. Instrum. Methods Phys. Res., Sect. A 285, 313 (1989).

[22] S. G. Anderson and J. B. Rosenzweig, Phys. Rev. ST Accel. Beams 3, 094201 (2000).

[23] K. Bane and P. Emma, in Proceedings of the 21st Particle Accelerator Conference, PAC2005, Knoxville, TN, 2005 (IEEE, Piscataway, NJ, 2005), p. 4266.

[24] K. Bane, in Workshop on the physics and applications of high brightness electron beams, Erice, Italy, 2005, Report No. SLAC-PUB-11829, 2006.

[25] Z. Huang, K. Bane, Y. Ding, and P. Emma, Phys. Rev. ST Accel. Beams 13, 092801 (2010).

[26] P. Emma et al., Phys. Rev. Lett. 112, 034801 (2014).

[27] M. Harrison et al., in Proceedings of the 25th Particle Accelerator Conference, PAC-2013, Pasadena, CA, 2013 (IEEE, New York, 2013).

[28] K. Bane and G. Stupakov, Nucl. Instrum. Methods Phys. Res., Sect. A 690, 106 (2012).

[29] K. Bane and G. Stupakov, Reports No. SLAC-PUB-15852, 2013 and No. LCLS-II TN-13-01. 